\title{
Eigenvalues of the volume operator in loop quantum gravity
}

\author{
Krzysztof A. Meissner ${ }^{1}$ \\ Institute of Theoretical Physics, Warsaw University \\ Hoża 69, 00-681 Warsaw, Poland \\ and \\ CERN, Theoretical Division
}

\begin{abstract}
We present a simple method to calculate certain sums of the eigenvalues of the volume operator in loop quantum gravity. We derive the asymptotic distribution of the eigenvalues in the classical limit of very large spins which turns out to be of a very simple form. The results can be useful for example in the statistical approach to quantum gravity.
\end{abstract}

\section{Introduction}

Volume operators were introduced by Rovelli and Smolin [1] and by Ashtekar and Lewandowski [2] (the latter will be used in the current paper). The volume operator is much less understood than the area operator, one of the reasons being that it is not diagonal in any reasonable basis and there are ambiguities in defining its action on a given graph (see 3. and references therein; clarification of the issue is given in [4]). The matrix elements of the volume operator were obtained in [5], 6] (in the context of cosmology [7]); however, the formulae are very complicated and it is extremely difficult to get analytic expressions for the eigenvalues of the volume operator, except for very small values of the momenta (for some bounds on the eigenvalues see [8]). Therefore it seems worthwhile to try to find some alternative way of describing the eigenvalues. In this paper we show that relatively simple formulae can be obtained for the sums of squares (or higher even powers) of the eigenvalues, with the most important "quantum numbers" in the vertex

\footnotetext{
${ }^{1}$ E-mail address: Krzysztof .Meissner@fuw.edu.pl
} 
fixed: $j, j_{1}, j_{2}, j_{3}$, where $j$ is the total spin and $j_{1}, j_{2}, j_{3}$ are spins of the incoming legs. We also calculate the sums of higher powers (4, 6 and 8$)$ of the eigenvalues with fixed $j_{1}, j_{2}, j_{3}$. At the end of the paper we derive (in the form of an integral) the distribution of the eigenvalues for fixed $j_{1}, j_{2}, j_{3}, j$ in the classical limit, when all the values of spins tend to infinity, and show that without fixing $j$ the integral can be performed; the result turns out to be surprisingly simple: $\rho(\lambda)=\arccos \left(\lambda /\left(j_{1} j_{2} j_{3}\right) /\left(2 j_{1} j_{2} j_{3}\right)\right.$ for $|\lambda| \leq j_{1} j_{2} j_{3}$ and $\rho(\lambda)=0$ otherwise.

\section{Volume operator}

The volume operator $V$ is defined as

$$
V=\sqrt{|\hat{q}|}
$$

where $\hat{q}$ is analogous to the determinant of the metric

$$
\hat{q}=\sum_{I<J<K} \epsilon_{i j k} J_{I}^{i} J_{J}^{j} J_{K}^{k}
$$

Here, $I, J, K$ run through $1,2, \ldots, N(N \geq 3)$ and $i, j, k$ are $S U(2)$ indices and we omit an overall constant proportional to the Planck volume. Eigenvalue of $V$ describes a contribution to the volume from a given vertex with $N+1$ legs with "spin conservation'. We will concentrate on the most important case $N=3$ (so called 4-valent vertex) and later we will comment on the application of the method to higher $N$.

The operator $\hat{q}$ has several important properties:

- It commutes with all $\mathbf{J}_{I}^{2}$

$$
\left[\hat{q}, \mathbf{J}_{I}^{2}\right]=0 . \quad I=1,2,3
$$

so we can treat in the following all $\mathbf{J}_{I}^{2}=j_{I}\left(j_{I}+1\right)$ as fixed.

- It commutes with all components of the total spin $\mathbf{J}$

$$
[\hat{q}, \mathbf{J}]=0 . \quad \mathbf{J}:=\mathbf{J}_{1}+\mathbf{J}_{2}+\mathbf{J}_{3}
$$

so its eigenvalues can be further indexed by the eigenvalues of $\mathbf{J}^{2}$, denoted by $j(j+1)$. Note that because of (41) the eigenvalues of $\hat{q}^{2}$ are the same for all $m$ in the $(j, m)$ multiplet. By this procedure, we are automatically in the gauge invariant case (where $\mathbf{J}_{4}:=-\mathbf{J}$ satisfies $\left.\mathbf{J}_{1}+\mathbf{J}_{2}+\mathbf{J}_{3}+\mathbf{J}_{4}=0\right)$. 
- operator $\hat{q}$ treats all four spins $\mathbf{J}_{1}, \mathbf{J}_{2}, \mathbf{J}_{3}, \mathbf{J}_{4}$ satisfying $\mathbf{J}_{1}+\mathbf{J}_{2}+\mathbf{J}_{3}+\mathbf{J}_{4}=$ 0 in the same way (modulo sign) since for example

$$
\hat{q}=\epsilon_{i j k} J_{1}^{i} J_{2}^{j} J_{3}^{k}=-\epsilon_{i j k} J_{1}^{i} J_{2}^{j} J_{4}^{k}
$$

- The operator $\hat{q}$ therefore suggests a natural way to number states in a vertex: we specify $j_{1}, j_{2}, j_{3}, j, m$ of incoming legs and the eigenvalue of $\hat{q}$ (the numbering is usually done in a "non-democratic" way: spins $j_{1}$ and $j_{2}$ are composed to spin $j$, spins $j_{3}$ and $j_{4}$ are composed also to spin $j$ and the two spins $j$ are composed to a singlet)

Since the operator $\hat{q}$ is self adjoint and (as can be easily seen from antisymmetry of $\epsilon_{i j k}$ and cyclic property of trace) trace of any odd power of $\hat{q}$ vanishes, therefore $\hat{q}$ has only real eigenvalues that are either 0 or come in pairs $\left(\lambda_{i},-\lambda_{i}\right)$. We assume everywhere that all $j_{I}$ are bigger than 0 since if any of $j_{I}$ is equal to 0 then all the eigenvalues of the volume operator are also equal to 0 .

As was shown in [5, [6] operator $\hat{q}$ has very complicated matrix elements as a function of $j, j_{1}, j_{2}, j_{3}$ (the matrix elements were obtained from the formula $\hat{q}=\mathrm{i}\left[\mathbf{J}_{1} \cdot \mathbf{J}_{2}, \mathbf{J}_{2} \cdot \mathbf{J}_{3}\right]$ and projection on a state with given $j$ ).

Note that there exists a convenient representation of the operator $\hat{q}$ in the space of polynomials of degree $\left(2 j_{1}+1\right)$ in $x,\left(2 j_{2}+1\right)$ in $y$ and $\left(2 j_{3}+1\right)$ in $z$ where

$$
\left|j_{1}, m_{1}\right\rangle=\sqrt{\left(2 j_{1}\right) ! /\left(j_{1}-m_{1}\right) ! /\left(j_{1}+m_{1}\right) !} x^{j_{1}+m_{1}}
$$

and the generators

$$
J_{1}^{+}=-x^{2} \partial_{x}+2 j_{1} x, \quad J_{1}^{-}=\partial_{x}, \quad J_{1}^{3}=x \partial_{x}-j_{1}
$$

and analogously for two other sets of generators. Then $\hat{q}$ has the form:

$$
\begin{aligned}
\hat{q}= & \frac{\mathrm{i}}{2}\left[-(x-y)(y-z)(z-x) \partial_{x} \partial_{y} \partial_{z}+j_{1}(y-z)(y+z-2 x) \partial_{y} \partial_{z}\right. \\
& +j_{2}(z-x)(z+x-2 y) \partial_{z} \partial_{x}+j_{3}(x-y)(x+y-2 z) \partial_{x} \partial_{y} \\
& \left.+2 j_{1} j_{2}(x-y) \partial_{z}+2 j_{2} j_{3}(y-z) \partial_{x}+2 j_{3} j_{1}(z-x) \partial_{y}\right]
\end{aligned}
$$

As we can see explicitly, the $\hat{q}$ operator conserves the total degree of the state (i.e. $m=m_{1}+m_{2}+m_{3}$ ) and indeed has complicated matrix elements especially after projection on eigenstates of $\mathbf{J}^{2}$. 


\section{The square of the determinant operator}

We consider the second power of the operator $\hat{q}$ :

$$
\hat{q}^{2}=\left(\epsilon_{i j k} J_{1}^{i} J_{2}^{j} J_{3}^{k}\right)^{2} .
$$

Using the well known formula

$$
\begin{aligned}
\epsilon_{i j k} \epsilon_{l m n}= & \delta_{i l} \delta_{j m} \delta_{k n}-\delta_{j l} \delta_{i m} \delta_{k n}+\delta_{k l} \delta_{i m} \delta_{j n} \\
& -\delta_{i l} \delta_{k m} \delta_{j n}+\delta_{j l} \delta_{k m} \delta_{i n}-\delta_{k l} \delta_{j m} \delta_{i n}
\end{aligned}
$$

we obtain

$$
\begin{aligned}
\hat{q}^{2}= & \mathbf{J}_{1}^{2} \mathbf{J}_{2}^{2} \mathbf{J}_{3}^{2}-\mathbf{J}_{1}^{2} J_{2}^{i}\left(\mathbf{J}_{2} \cdot \mathbf{J}_{3}\right) J_{3}^{i}-\mathbf{J}_{3}^{2} J_{1}^{i}\left(\mathbf{J}_{1} \cdot \mathbf{J}_{2}\right) J_{2}^{i}-\mathbf{J}_{2}^{2} J_{1}^{i}\left(\mathbf{J}_{1} \cdot \mathbf{J}_{3}\right) J_{3}^{i} \\
& +J_{1}^{i}\left(\mathbf{J}_{1} \cdot \mathbf{J}_{2}\right)\left(\mathbf{J}_{2} \cdot \mathbf{J}_{3}\right) J_{3}^{i}+J_{1}^{i} J_{2}^{j}\left(\mathbf{J}_{1} \cdot \mathbf{J}_{3}\right) J_{2}^{i} J_{3}^{j}
\end{aligned}
$$

We want to trace the operator with the states

$$
\left|\left(j_{1}, m_{1}\right) \otimes\left(j_{2}, m_{2}\right) \otimes\left(j_{3}, m_{3}\right)\right\rangle
$$

(often written as $\left|m_{1}, m_{2}, m_{3}\right\rangle$ for short), not yet imposing any restriction on $j$ and $m=m_{1}+m_{2}+m_{3}$. The calculation uses well known formulae (straightforward from (6) and (7)):

$$
\begin{aligned}
J_{I}^{+}\left|j_{I}, m_{I}\right\rangle & =\sqrt{j_{I}\left(j_{I}+1\right)-m_{I}\left(m_{I}+1\right)}\left|j_{I}, m_{I}+1\right\rangle \\
J_{I}^{-}\left|j_{I}, m_{I}\right\rangle & =\sqrt{j_{I}\left(j_{I}+1\right)-m_{I}\left(m_{I}-1\right)}\left|j_{I}, m_{I}-1\right\rangle \\
J_{I}^{3}\left|j_{I}, m_{I}\right\rangle & =m_{I}\left|j_{I}, m_{I}\right\rangle
\end{aligned}
$$

The result reads

$$
\begin{aligned}
& \left\langle m_{1}, m_{2}, m_{3}\left|\hat{q}^{2}\right| m_{1}, m_{2}, m_{3}\right\rangle= \\
& \quad \frac{1}{2} m_{1}^{2}\left[\left(j_{2}\left(j_{2}+1\right)-m_{2}^{2}\right)\left(j_{3}\left(j_{3}+1\right)-m_{3}^{2}\right)-m_{2} m_{3}\right] \\
& \quad+\frac{1}{2} m_{2}^{2}\left[\left(j_{1}\left(j_{1}+1\right)-m_{1}^{2}\right)\left(j_{3}\left(j_{3}+1\right)-m_{3}^{2}\right)-m_{1} m_{3}\right] \\
& \quad+\frac{1}{2} m_{3}^{2}\left[\left(j_{1}\left(j_{1}+1\right)-m_{1}^{2}\right)\left(j_{2}\left(j_{2}+1\right)-m_{2}^{2}\right)-m_{1} m_{2}\right] .
\end{aligned}
$$

Summing over all $m_{1}, m_{2}, m_{3}$, we have the result

$$
\operatorname{Tr} \hat{q}^{2}=\frac{2}{9} j_{1}\left(j_{1}+1\right)\left(2 j_{1}+1\right) j_{2}\left(j_{2}+1\right)\left(2 j_{2}+1\right) j_{3}\left(j_{3}+1\right)\left(2 j_{3}+1\right) \text {. }
$$


Since the total number of states is equal to $\left(2 j_{1}+1\right)\left(2 j_{2}+1\right)\left(2 j_{3}+1\right)$ the average eigenvalue of $\hat{q}^{2}$ is equal to

$$
\left\langle\hat{q}^{2}\right\rangle=\frac{2}{9} j_{1}\left(j_{1}+1\right) j_{2}\left(j_{2}+1\right) j_{3}\left(j_{3}+1\right) .
$$

In the last section we will derive the general formula for $\left\langle\hat{q}^{2 N}\right\rangle$ in the limit of large $j$ 's.

\section{Sum of the eigenvalues with fixed $j$}

In this section we calculate $\operatorname{Tr} \hat{q}^{2}$ with an additional condition

$$
m_{1}+m_{2}+m_{3}=m,
$$

where to be definite we take $m \leq 0$. We sum in this way many different $j$ multiplets with $j \geq-m$; it is important to notice that because of (3) the eigenvalues of $\hat{q}^{2}$ are the same for all $(j, m)$ components of a given $j$ multiplet.

As one can easily convince oneself it is a non-trivial problem to impose the condition (17) directly. Therefore we use an indirect method that will partly do the bookkeeping for us: we perform the sum with the additional factor

$$
f_{2}\left(w, j_{1}, j_{2}, j_{3}\right):=\operatorname{Tr}\left(\hat{q}^{2} w^{J_{1}^{3}+J_{2}^{3}+J_{3}^{3}}\right)
$$

and at the end we will get to the final result by picking up the coefficient in front of $w^{m}$.

We use the formula

$$
\sum_{m_{1}=-j_{1}}^{j_{1}} m_{1}^{p} w^{m_{1}}=\left(w \partial_{w}\right)^{p} \frac{w^{-j_{1}}-w^{j_{1}+1}}{1-w} .
$$

The computation of $f_{2}\left(w, j_{1}, j_{2}, j_{3}\right)$ from (14) gives

$$
\begin{aligned}
& (1-w) f_{2}\left(w, j_{1}, j_{2}, j_{3}\right)=\frac{2}{(1-w)^{8}} w^{-j_{1}-j_{2}-j_{3}+1}\left\{j_{1} j_{2} j_{3}\left(j_{1}+j_{2}+j_{3}\right)\right. \\
& -w\left[j_{1} j_{2}\left(\left(j_{1}+j_{2}\right)\left(2 j_{3}+1\right)+2 j_{3}-1\right)+(1 \leftrightarrow 3)+(2 \leftrightarrow 3)\right] \\
& +w^{2}\left[\left(j_{1}+1\right)\left(j_{1}+\frac{3}{2}\left(j_{2}+j_{3}\right)\left(2 j_{1}-1\right)+6 j_{1} j_{2} j_{3}\right)+(1 \leftrightarrow 2)+(1 \leftrightarrow 3)\right] \\
& -w^{3}\left[\left(j_{1}+1\right)\left(j_{2}+1\right)\left(\left(j_{1}+j_{2}\right)\left(2 j_{3}+1\right)+2 j_{3}-1\right)+(1 \leftrightarrow 3)+(2 \leftrightarrow 3)\right]
\end{aligned}
$$




$$
\begin{aligned}
& \left.+w^{4}\left(j_{1}+1\right)\left(j_{2}+1\right)\left(j_{3}+1\right)\left(j_{1}+j_{2}+j_{3}+3\right)\right\} \\
& -\left(j_{1} \rightarrow-j_{1}-1\right)-\left(j_{2} \rightarrow-j_{2}-1\right)-\left(j_{3} \rightarrow-j_{3}-1\right) \\
& +\left(j_{1} \rightarrow-j_{1}-1, j_{2} \rightarrow-j_{2}-1\right)+\left(j_{2} \rightarrow-j_{2}-1, j_{3} \rightarrow-j_{3}-1\right) \\
& +\left(j_{1} \rightarrow-j_{1}-1, j_{3} \rightarrow-j_{3}-1\right) \\
& -\left(j_{1} \rightarrow-j_{1}-1, j_{2} \rightarrow-j_{2}-1, j_{3} \rightarrow-j_{3}-1\right) .
\end{aligned}
$$

To obtain the sum of squares of eigenvalues with fixed $j$ we subtract the result for $f_{2}\left(w, j_{1}, j_{2}, j_{3}\right)$ with $m=-j$ from the result for $m=-j-1$ (it follows from the fact that the eigenvalues are the same for all $m$ in $(j, m)$ multiplets). We therefore expand $(1-w) f_{2}\left(w, j_{1}, j_{2}, j_{3}\right)$ given in (20) in $w$, and the coefficient in front of $w^{-j}$ is the desired sum of squares of eigenvalues with fixed $j$. Hence for example:

- the eigenvalue for $j=\left(j_{1}+j_{2}+j_{3}\right)$ is equal to 0

- for $j=\left(j_{1}+j_{2}+j_{3}-1\right)$ eigenvalues are equal to $\pm \sqrt{j_{1} j_{2} j_{3}\left(j_{1}+j_{2}+j_{3}\right)}$

- for $j=\left(j_{1}+j_{2}+j_{3}-2\right)$ there is an eigenvalue 0 and two eigenvalues equal to $\pm \sqrt{\left(4 j_{1} j_{2} j_{3}-j_{1} j_{2}-j_{2} j_{3}-j_{1} j_{3}\right)\left(j_{1}+j_{2}+j_{3}-1\right)+j_{1} j_{2} j_{3}}$ (if any of the $j_{I}$ is equal to $1 / 2$ then some of these eigenvalues may be missing).

We may note that for the $n$-valent vertex $(n>4)$ the method works in exactly the same way: in the $\hat{q}^{2}$ operator, beyond the already discussed terms, we encounter also terms of the form

$$
\epsilon_{i j k} J_{1}^{i} J_{2}^{j} J_{3}^{k} \epsilon_{l m n} J_{1}^{l} J_{2}^{m} J_{4}^{n}
$$

After sandwiching between $\left|m_{1}, m_{2}, m_{3}, m_{4}\right\rangle$ we have to assign $k=3, n=3$ and therefore $(i, j, k, l)=(+,-,-,+)$ or $(i, j, k, l)=(-,+,+,-)$, so that the appropriate term in $f_{2}\left(w, j_{1}, j_{2}, j_{3}, j_{4}\right)$ reads

$$
\sum_{m_{1}, m_{2}, m_{3}, m_{4}} w^{m_{1}+m_{2}+m_{3}+m_{4}} \frac{1}{2} m_{3} m_{4}\left(j_{1}\left(j_{1}+1\right)-m_{1}^{2}\right)\left(j_{2}\left(j_{2}+1\right)-m_{2}^{2}\right)
$$

and the sum can be performed without difficulty.

As can be easily seen the terms of the form $\epsilon_{i j k} J_{1}^{i} J_{2}^{j} J_{3}^{k} \epsilon_{l m n} J_{1}^{l} J_{4}^{m} J_{5}^{n}$ or $\epsilon_{i j k} J_{1}^{i} J_{2}^{j} J_{3}^{k} \epsilon_{l m n} J_{4}^{l} J_{5}^{m} J_{6}^{n}$ give no contribution to $f_{2}$. 
We have also calculated along the same lines

$$
f_{4}\left(w, j_{1}, j_{2}, j_{3}\right):=\operatorname{Tr}\left(\hat{q}^{4} w^{J_{1}^{3}+J_{2}^{3}+J_{3}^{3}}\right)
$$

but the resulting formulae are too long to be presented here.

\section{The density distribution for eigenvalues}

It would be very desirable to have also higher-order moments,

$$
\left.\operatorname{Tr} \hat{q}^{2 n}\right|_{j, j_{1}, j_{2}, j_{3}},
$$

since we could then reconstruct the full density by inverse Mellin transform of the relation

$$
\left.\operatorname{Tr} \hat{q}^{2 n}\right|_{j, j_{1}, j_{2}, j_{3}}=\int \mathrm{d} \lambda \rho_{j}(\lambda) \lambda^{2 n} .
$$

Since $\hat{q}$ is an operator in a finite-dimensional space, the density distribution is just a sum of Dirac delta functions; we aim at finding a smooth approximation of this distribution for large values of the spins involved.

In this section we will calculate higher moments $\left(\operatorname{Tr} \hat{q}^{4}, \operatorname{Tr} \hat{q}^{6}\right.$ and $\left.\operatorname{Tr} \hat{q}^{8}\right)$ as a function of $j_{1}, j_{2}$, and $j_{3}$, but with $j$ not fixed.

Introducing the notation

$$
\langle B\rangle:=\frac{\operatorname{Tr} B}{\operatorname{Tr} \mathbf{1}},
$$

we have the relation

$$
\begin{aligned}
\left\langle e^{-A}\right\rangle= & \exp \left(-\langle A\rangle+\frac{1}{2}\left(\left\langle A^{2}\right\rangle-\langle A\rangle^{2}\right)\right. \\
& -\frac{1}{6}\left(\left\langle A^{3}\right\rangle-3\left\langle A^{2}\right\rangle\langle A\rangle+2\langle A\rangle^{3}\right) \\
& \left.+\frac{1}{24}\left(\left\langle A^{4}\right\rangle-4\left\langle A^{3}\right\rangle\langle A\rangle-3\left\langle A^{2}\right\rangle^{2}+12\left\langle A^{2}\right\rangle\langle A\rangle^{2}-6\langle A\rangle^{4}\right)+\ldots\right) .
\end{aligned}
$$

Neglecting terms of the third order and higher in the exponent (Gaussian approximation) we have

$$
\left\langle e^{-\alpha \hat{q}}\right\rangle \approx e^{-\langle\alpha \hat{q}\rangle+\frac{\alpha^{2}}{2}\left(\left\langle\hat{q}^{2}\right\rangle-\langle\hat{q}\rangle^{2}\right)}=\int \mathrm{d} \lambda \rho_{G}(\lambda) e^{-\alpha \lambda}
$$


and since $\langle\hat{q}\rangle=0$ we have

$$
\rho_{G}(\lambda)=\sqrt{\frac{1}{2 \pi \beta}} e^{-\lambda^{2} /(2 \beta)},
$$

where

$$
\beta=\left\langle\hat{q}^{2}\right\rangle
$$

In the Gaussian approximation

$$
\left\langle\hat{q}^{2 n}\right\rangle=(2 n-1) ! !\left\langle\hat{q}^{2}\right\rangle^{n}
$$

As we already know,

$$
\left\langle\hat{q}^{2}\right\rangle=\frac{2}{9} F_{3}
$$

where we introduced

$$
\begin{aligned}
& F_{0}=1 \\
& F_{1}=j_{1}\left(j_{1}+1\right)+j_{2}\left(j_{2}+1\right)+j_{3}\left(j_{3}+1\right), \\
& F_{2}=j_{1}\left(j_{1}+1\right) j_{2}\left(j_{2}+1\right)+j_{1}\left(j_{1}+1\right) j_{3}\left(j_{3}+1\right)+j_{2}\left(j_{2}+1\right) j_{3}\left(j_{3}+1\right), \\
& F_{3}=j_{1}\left(j_{1}+1\right) j_{2}\left(j_{2}+1\right) j_{3}\left(j_{3}+1\right) .
\end{aligned}
$$

Using the form (11) we obtain (with the help of the MAPLE program)

$$
\begin{gathered}
\left\langle\hat{q}^{4}\right\rangle=\frac{F_{3}}{225}\left(24 F_{3}-8 F_{2}+11 F_{1}-12 F_{0}\right), \\
\left\langle\hat{q}^{6}\right\rangle=\frac{F_{3}}{22050}\left(1440 F_{3}^{2}-1440 F_{3} F_{2}+480 F_{2}^{2}+2538 F_{3} F_{1}-1656 F_{2} F_{1}\right. \\
\left.-6909 F_{3} F_{0}+1042 F_{1}^{2}+5158 F_{2} F_{0}-4815 F_{1} F_{0}+3222 F_{0}^{2}\right)(35)
\end{gathered}
$$

and

$$
\begin{aligned}
\left\langle\hat{q}^{8}\right\rangle= & \frac{F_{3}}{99225}\left(4480 F_{3}^{3}-8960 F_{3}^{2} F_{2}+8064 F_{3} F_{2}^{2}+19936 F_{3}^{2} F_{1}\right. \\
& -78032 F_{3}^{2} F_{0}-2688 F_{2}^{3}-38304 F_{3} F_{2} F_{1}+180204 F_{3} F_{2} \\
& +41853 F_{3} F_{1}^{2}+18480 F_{2}^{2} F_{1}-385701 F_{3} F_{1} F_{0}-91932 F_{2}^{2} F_{0} \\
& -36351 F_{2} F_{1}^{2}+691740 F_{3} F_{0}^{2}+329841 F_{2} F_{1} F_{0}+18081 F_{1}^{3} \\
& \left.-549288 F_{2} F_{0}^{2}-204552 F_{1}^{2} F_{0}+521316 F_{1} F_{0}^{2}-285282 F_{0}^{3}\right) .
\end{aligned}
$$


The result for $\left\langle\hat{q}^{2 n}\right\rangle$ has the form $F_{3} \cdot P_{n-1}$ where $P_{n-1}$ is a polynomial with alternating signs, homogeneous of degree $(n-1)$ in $F_{3}, F_{2}, F_{1}, F_{0}$.

We were unable to find a general expression for the coefficients of these polynomials, however, as will be shown in the next section, we were able to prove that for $\left\langle\hat{q}^{2 n}\right\rangle$ the coefficient in front of $F_{3}^{n}$, which is the most important

term for large spins, is equal to $\frac{(2 n) ! !}{(2 n+1)(2 n+1) ! !}$ and for $n>1$ it differs from the Gaussian approximation result $(2 n-1) ! !(2 / 9)^{n}$.

\section{Classical distribution of the eigenvalues}

In this section we will find the classical distribution (both integrated $\rho(\lambda)$ and in the subspace of fixed total spin $\rho_{j}(\lambda)$ ) of the eigenvalues of the operator $\hat{q}$ in the limit of large spins. In this limit we can disregard any commutators of $\mathbf{J}_{I}$ with itself (since they introduce lower power of spins) and the case boils down to the classical density.

For the classical density we have,

$$
\begin{aligned}
\int_{-j_{1} j_{2} j_{3}}^{j_{1} j_{2} j_{3}} \mathrm{~d} \lambda \rho_{j}(\lambda) \lambda^{2 n}= & N^{-1} \int \frac{\mathrm{d} \Omega_{1} \mathrm{~d} \Omega_{2} \mathrm{~d} \Omega_{3}}{(4 \pi)^{3}}\left(\left(\mathbf{J}_{1} \times \mathbf{J}_{2}\right) \cdot \mathbf{J}_{3}\right)^{2 n} . \\
& \delta\left(\left(\mathbf{J}_{1}+\mathbf{J}_{2}+\mathbf{J}_{3}\right)^{2}-j^{2}\right),
\end{aligned}
$$

where we integrate over angular positions of $\mathbf{J}_{1}, \mathbf{J}_{2}$ and $\mathbf{J}_{3}$, and $N$ is a normalization factor:

$$
N=\int \frac{\mathrm{d} \Omega_{1} \mathrm{~d} \Omega_{2} \mathrm{~d} \Omega_{3}}{(4 \pi)^{3}} \delta\left(\left(\mathbf{J}_{1}+\mathbf{J}_{2}+\mathbf{J}_{3}\right)^{2}-j^{2}\right) .
$$

We introduce also the integrated density satisfying

$$
\int_{-j_{1} j_{2} j_{3}}^{j_{1} j_{2} j_{3}} \mathrm{~d} \lambda \rho(\lambda) \lambda^{2 n}=\int \frac{\mathrm{d} \Omega_{1} \mathrm{~d} \Omega_{2} \mathrm{~d} \Omega_{3}}{(4 \pi)^{3}}\left(\left(\mathbf{J}_{1} \times \mathbf{J}_{2}\right) \cdot \mathbf{J}_{3}\right)^{2 n} .
$$

Since only relative angles are important, we can fix some positions:

$$
\theta_{3}=0, \quad \phi_{2}=0
$$

and then for example

$$
\left(\mathbf{J}_{1} \times \mathbf{J}_{2}\right) \cdot \mathbf{J}_{3}=j_{1} j_{2} j_{3} \sin \theta_{1} \sin \theta_{2} \sin \phi .
$$


We introduce coordinates on $S^{3}$ :

$$
\begin{aligned}
& x=\sin \theta_{1} \sin \theta_{2} \sin \phi, \\
& y=\sin \theta_{1} \sin \theta_{2} \cos \phi, \\
& z=\sin \theta_{1} \cos \theta_{2}, \\
& t=\cos \theta_{1} .
\end{aligned}
$$

Then

$$
\begin{aligned}
\int_{-j_{1} j_{2} j_{3}}^{j_{1} j_{2} j_{3}} \mathrm{~d} \lambda \rho_{j}(\lambda) \lambda^{2 n}= & \frac{1}{8 \pi N} \int \frac{\mathrm{d} x \mathrm{~d} y \mathrm{~d} z \mathrm{~d} t}{\sqrt{1-t^{2}}} 2 \delta\left(x^{2}+y^{2}+z^{2}+t^{2}-1\right) \\
& \left(j_{1} j_{2} j_{3} x\right)^{2 n} \delta\left(j_{1}^{2}+j_{2}^{2}+j_{3}^{2}+2 j_{1} j_{2} t+2 j_{1} j_{3} \frac{z}{\sqrt{1-t^{2}}}\right. \\
& \left.+2 j_{2} j_{3} \frac{z t}{\sqrt{1-t^{2}}}+2 j_{2} j_{3} y-j^{2}\right)
\end{aligned}
$$

Comparing the sides we obtain the final result, i.e. the classical distribution function of eigenvalues in terms of $j_{1}, j_{2}, j_{3}, j$ :

$$
\begin{aligned}
\rho_{j}(\lambda)= & \frac{1}{8 \pi N j_{1} j_{2} j_{3}} \int \frac{\mathrm{d} y \mathrm{~d} z \mathrm{~d} t}{\sqrt{1-t^{2}}} 2 \delta\left(\tilde{\lambda}^{2}+y^{2}+z^{2}+t^{2}-1\right) \\
& \delta\left(j_{1}^{2}+j_{2}^{2}+j_{3}^{2}+2 j_{1} j_{2} t+2 j_{1} j_{3} \frac{z}{\sqrt{1-t^{2}}}\right. \\
& \left.+2 j_{2} j_{3} \frac{z t}{\sqrt{1-t^{2}}}+2 j_{2} j_{3} y-j^{2}\right),
\end{aligned}
$$

where

$$
\tilde{\lambda}=\lambda /\left(j_{1} j_{2} j_{3}\right)
$$

We can integrate over $t$ :

$$
\begin{aligned}
\rho_{j}(\lambda)= & \frac{1}{8 \pi N j_{1} j_{2} j_{3}} \int_{0}^{\sqrt{1-\tilde{\lambda}^{2}}} \int_{0}^{2 \pi} \frac{r \mathrm{~d} r \mathrm{~d} \phi}{\sqrt{r^{2}+\tilde{\lambda}^{2}} \sqrt{1-r^{2}-\tilde{\lambda}^{2}}} \\
& \sum_{\epsilon= \pm 1} \delta\left(j_{1}^{2}+j_{2}^{2}+j_{3}^{2}+2 j_{1} j_{2} \epsilon \sqrt{1-r^{2}-\tilde{\lambda}^{2}}+2 j_{1} j_{3} \frac{r \sin \phi}{\sqrt{r^{2}+\tilde{\lambda}^{2}}}\right. \\
& \left.+2 j_{2} j_{3} \epsilon \frac{r \sin \phi \sqrt{1-r^{2}-\tilde{\lambda}^{2}}}{\sqrt{r^{2}+\tilde{\lambda}^{2}}}+2 j_{2} j_{3} r \cos \phi-j^{2}\right) .
\end{aligned}
$$


It is difficult to perform the integral in this form. However, performing the same steps for the integrated distribution function we obtain a surprisingly simple result:

$$
\rho(\lambda)=\frac{1}{2 j_{1} j_{2} j_{3}} \int_{0}^{\sqrt{1-\tilde{\lambda}^{2}}} \frac{r \mathrm{~d} r}{\sqrt{r^{2}+\tilde{\lambda}^{2}} \sqrt{1-r^{2}-\tilde{\lambda}^{2}}}=\frac{1}{2 j_{1} j_{2} j_{3}} \arccos (\tilde{\lambda})
$$

for $|\tilde{\lambda}| \leq 1$ and 0 for $|\tilde{\lambda}|>1$.

Therefore we get the coefficient in front of $F_{3}^{n}$ for the integrated trace

$$
\left\langle\hat{q}^{2 n}\right\rangle \rightarrow \int_{-1}^{1} \mathrm{~d} x \frac{1}{2} \arccos (x) x^{2 n} F_{3}^{n}=\frac{(2 n) ! !}{(2 n+1)(2 n+1) ! !} F_{3}^{n} .
$$

Hence for example we recover the leading coefficients of (321), (34), (355) and (36):

$$
\left\langle\hat{q}^{2}\right\rangle \rightarrow \frac{2}{9} F_{3}, \quad\left\langle\hat{q}^{4}\right\rangle \rightarrow \frac{8}{75} F_{3}^{2}, \quad\left\langle\hat{q}^{6}\right\rangle \rightarrow \frac{16}{245} F_{3}^{3}, \quad\left\langle\hat{q}^{8}\right\rangle \rightarrow \frac{128}{2835} F_{3}^{4} .
$$

Concluding, by using the methods described in this paper we can learn something about the distribution of the eigenvalues of the volume operator in the quantum case, and there exists an explicit formula for this distribution in the limiting case of large spins, i.e. the classical case. It would be interesting to compare these results with the numerical analysis for low spins (up to 50) given in [5].

Besides being mathematically interesting, these results can be important for example in the statistical approach to loop quantum gravity.

Several conceptual problems arise in this context by the results of the present paper. The first one is what observables (besides the volume) should be specified to actually describe a macroscopic state in a given region. Since the contribution to the volume from a given 4 -valent vertex grows like $j_{1} \cdot j_{2} \cdot j_{3}$ it is important to know whether these observables provide suppression of the contribution from very large spins rendering final expectation value of the volume finite. The second problem is connected with the first one and concerns the bulk entropy: there are many sets of graphs with many different assignments of spins on legs that give (macroscopically) the same volume. If we specify both the volume and the other observables we are left with a certain number of graphs. The question is, whether the logarithm of this number is connected with the bulk entropy much as sequences of spins are connected with the black hole surface entropy [9]. 


\section{Acknowledgements}

The author would like to thank Hermann Nicolai and the whole group at the AEI for hospitality. Discussions with Abhay Ashtekar, Johannes Brunnemann, Thomas Thiemann and especially Jurek Lewandowski are gratefully acknowledged. This work was partially supported by the Polish KBN grant 2P03B 00125 and the European Program HPRN-CT-2002-0277.

\section{References}

[1] C. Rovelli and L. Smolin, "Discreteness of area and volume in quantum gravity", Nucl. Phys. B442 (1995) 593; Erratum, Nucl. Phys. B456 (1995) 734 .

[2] A. Ashtekar and J. Lewandowski, "Differential geometry on the space of connections via graphs and projective limits", J. Geom. Phys. 17 (1995) 191;

"Quantum theory of geometry, II: Volume operators", Adv. Theor. Math. Phys. 1 (1998) 388, gr-qc/9711031.

[3] A. Ashtekar and J. Lewandowski, "Background independent quantum gravity: A status report," Class. Quant. Grav. 21 (2004) R53, gr-qc/0404018

[4] K. Giesel and T. Thiemann, Consistency Check on Volume and Triad Operator Quantisation in Loop Quantum Gravity, gr-qc/0507036, gr-qc/0507037,

[5] T. Thiemann, "Closed formula for the matrix elements of the volume operator in canonical quantum gravity", J. Math. Phys. 39 (1998) 3347, gr-qc/9606091,

J. Brunnemann and T. Thiemann, 'Simplification of the spectral analysis of the volume operator in loop quantum gravity", gr-qc/0405060.

[6] R. De Pietri and C. Rovelli, "Geometry eigenvalues and scalar product from recoupling theory in loop quantum gravity," Phys. Rev. D 54 (1996) 2664, gr-qc/9602023.

[7] M. Bojowald, "Loop quantum cosmology. II: Volume operators", Class. Quant. Grav. 17 (2000) 1509, gr-qc/9910104. 
[8] S. A. Major and M. D. Seifert, "Modelling space with an atom of quantum geometry," Class. Quant. Grav. 19, 2211 (2002) gr-qc/0109056.

[9] A. Ashtekar, J. Baez, A. Corichi and K. Krasnov, "Quantum geometry and black hole entropy", Phys. Rev. Lett. 80 (1998) 904, gr-qc/9710007

M. Domagala and J. Lewandowski, Class. Quant. Grav. 21 (2004) 5233, gr-qc/0407051

K. A. Meissner, "Black hole entropy in loop quantum gravity," Class. Quant. Grav. 21 (2004) 5245, gr-qc/0407052. 